\title{
Is goal-directed fluid therapy based on dynamic variables alone sufficient to improve clinical outcomes among patients undergoing surgery? A meta-analysis
}

Qi-Wen Deng ${ }^{1 \dagger}$, Wen-Cheng Tan ${ }^{2+}$, Bing-Cheng Zhao ${ }^{3 \dagger}$, Shi-Hong Wen ${ }^{1}$, Jian-Tong Shen ${ }^{1}$ and Miao Xu ${ }^{1 *}$

\begin{abstract}
Background: Whether goal-directed fluid therapy based on dynamic predictors of fluid responsiveness (GDFTdyn) alone improves clinical outcomes in comparison with standard fluid therapy among patients undergoing surgery remains unclear.

Methods: PubMed, EMBASE, the Cochrane Library and ClinicalTrials.gov were searched for relevant studies. Studies comparing the effects of GDFTdyn with that of standard fluid therapy on clinical outcomes among adult patients undergoing surgery were considered eligible. Two analyses were performed separately: GDFTdyn alone versus standard fluid therapy and GDFTdyn with other optimization goals versus standard fluid therapy. The primary outcomes were short-term mortality and overall morbidity, while the secondary outcomes were serum lactate concentration, organ-specific morbidity, and length of stay in the intensive care unit (ICU) and in hospital.
\end{abstract}

Results: We included 37 studies with 2910 patients. Although GDFTdyn alone lowered serum lactate concentration (mean difference (MD) $-0.21 \mathrm{mmol} / \mathrm{L}, 95 \%$ confidence interval $(C \mathrm{l})(-0.39,-0.03), P=0.02)$, no significant difference was found between groups in short-term mortality (odds ratio (OR) $0.85,95 \% \mathrm{Cl}(0.32,2.24), P=0.74$ ), overall morbidity (OR $1.03,95 \% \mathrm{Cl}(0.31,3.37), P=0.97)$, organ-specific morbidity, or length of stay in the ICU and in hospital. Analysis of trials involving the combination of GDFTdyn and other optimization goals (mainly cardiac output (CO) or cardiac index (Clx)) showed a significant reduction in short-term mortality $(\mathrm{OR} 0.45,95 \% \mathrm{Cl}(0.24,0.85)$, $P=0.01)$, overall morbidity (OR $0.41,95 \% \mathrm{Cl}(0.28,0.58), P<0.00001)$, serum lactate concentration $(\mathrm{MD}-0.60 \mathrm{mmol} / \mathrm{L}$, $95 \% \mathrm{Cl}(-1.04,-0.15), P=0.009)$, cardiopulmonary complications (cardiac arrhythmia (OR $0.58,95 \% \mathrm{Cl}(0.37,0.92)$, $P=0.02)$, myocardial infarction $(\mathrm{OR} 0.35,95 \% \mathrm{Cl}(0.16,0.76), P=0.008)$, heart failure/cardiovascular dysfunction (OR $0.31,95 \% \mathrm{Cl}(0.14,0.67), P=0.003)$, acute lung injury/acute respiratory distress syndrome (OR $0.13,95 \% \mathrm{Cl}(0.02,0.74)$, $P=0.02)$, pneumonia (OR $0.4,95 \% \mathrm{Cl}(0.24,0.65), P=0.0002)$ ), length of stay in the ICU (MD -0.77 days, $95 \% \mathrm{Cl}$ $(-1.07,-0.46), P<0.00001)$ and in hospital (MD -1.18 days, $95 \% \mathrm{Cl}(-1.90,-0.46), P=0.001)$.

Conclusions: It was not the optimization of fluid responsiveness by GDFTdyn alone but rather the optimization of tissue and organ perfusion by GDFTdyn and other optimization goals that benefited patients undergoing surgery. Patients managed with the combination of GDFTdyn and $\mathrm{CO} / \mathrm{Cl}$ goals might derive most benefit.

Keywords: Goal-directed fluid therapy, Dynamic variables, Cardiac output, Surgery

\footnotetext{
* Correspondence: spring_xum@163.com

${ }^{\dagger}$ Qi-Wen Deng, Wen-Cheng Tan and Bing-Cheng Zhao contributed equally

to this work.

${ }^{1}$ Department of Anesthesiology, the First Affiliated Hospital, Sun Yat-sen

University, No.58, Zhongshan 2nd Road, Guangzhou 510080, China

Full list of author information is available at the end of the article
}

(c) The Author(s). 2018 Open Access This article is distributed under the terms of the Creative Commons Attribution 4.0 International License (http://creativecommons.org/licenses/by/4.0/), which permits unrestricted use, distribution, and reproduction in any medium, provided you give appropriate credit to the original author(s) and the source, provide a link to the Creative Commons license, and indicate if changes were made. The Creative Commons Public Domain Dedication waiver (http://creativecommons.org/publicdomain/zero/1.0/) applies to the data made available in this article, unless otherwise stated. 


\section{Background}

Inappropriate fluid administration in the intraoperative period is associated with a risk of hypovolemia or overload. It then causes tissue hypoxia and postoperative organ dysfunction. The postoperative complications have a huge impact on short-term and long-term mortality. The occurrence of these complications could reduce median survival by $69 \%$ [1]. Moreover, the increased morbidity and mortality is associated with a high healthcare cost [2]. Correcting tissue hypoxia is a crucial step to improve the prognosis of patients undergoing surgery.

Occult tissue hypoxia still occurs despite the normalization of standard physiologic variables, such as heart rate, blood pressure, central venous pressure (CVP) and urine output [3, 4]. Goal-directed fluid therapy based on dynamic variables (GDFTdyn) is defined as a spectrum of fluid management strategies reaching optimal preload by monitoring variables derived from cardiorespiratory interaction. These variables include stroke volume variation (SVV), systolic pressure variation (SPV), pulse pressure variation (PPV) and pleth variability index (PVI). They have emerged to target tissue perfusion in recent years. They are believed to be the markers of positions on the Frank-Starling curve, which are proportional to the degree of preload dependency. Compared with stroke volume optimization requiring quantification of the percentage change in stroke volume or oxygen delivery optimization requiring frequent calculations of oxygen delivery related variables, GDFTdyn is perceived to be more direct and less time-consuming. It is thought to be more convenient for healthcare providers to know whether a patient is a fluid responder or not. Moreover, as arterial cannulation and pulse oximeter are routinely used in moderate to high-risk patients undergoing surgery, these dynamic variables are easy to obtain and well-tolerated by patients. These advantages of GDFTdyn make it possible to be widely used in clinical practice.

Numerous clinical trials and systematic reviews have evaluated the efficacy and safety of GDFTdyn in patients undergoing surgery [5-9]. However, most of these clinical trials are of small sample size and the results of them contradict each other. On the other hand, there may be significant heterogeneity and methodological flaws in the previous meta-analyses. Especially, existing meta-analyses have failed to account for nonuniform application of other combined optimization goals in the GDFTdyn arms. These combined optimization goals are variables not derived from cardiorespiratory interaction, such as variables of flow, cardiac output (CO) or cardiac index (CIx). They might serve to confound the final results. As a result, whether GDFTdyn alone improves clinical outcomes among patients undergoing surgery or not remains uncertain.

Therefore, we performed the meta-analysis to determine the effects of GDFTdyn in comparison with standard fluid therapy on clinical outcomes among adult patients undergoing surgery. Especially, we compared GDFTdyn alone and GDFTdyn with other optimization goals separately to better address the question.

\section{Methods}

The meta-analysis was conducted following the recommendations of Cochrane Handbook for Systematic Reviews of Interventions [10], and reported following Preferred Reporting Items for Systematic Reviews and Meta-Analyses (PRISMA) guidelines [11] (see Additional file 1). The protocol of the study has been registered in PROSPERO (CRD42018106439).

\section{Literature search}

A systematic search of PubMed, EMBASE, the Cochrane Library and ClinicalTrials.gov was performed independently by two authors (QWD and WCT) to identify relevant studies in any language published from inception to 1 September 2018. Electronic search keywords were goal directed (goal targeted, goal oriented), and fluid management (fluid optimization, fluid therapy), surgery (operation, intraoperative, perioperative). Additional studies were identified by reviewing the reference lists of previous systematic reviews. The search strategy used in PubMed was as follow: (1) "goal directed"; (2) "goal targeted"; (3) "goal oriented"; (4) 1 or 2 or 3; (5) fluid; (6) hemodynamic; (7) haemodynamic; (8) 5 or 6 or 7; (9) management; (10) optimization; (11) therapy; (12) 9 or 10 or 11 ; (13) 8 and 12; (14) surg*; (15) operat"; (16) intraoperative*; (17) peri-

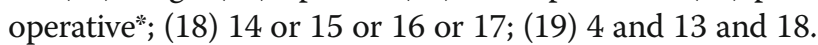

\section{Study selection}

After excluding studies based on title and abstract screening, two authors (QWD and BCZ) independently reviewed the full texts of the remaining studies. Consensus was resolved by the third author (WCT) when disagreement occurred. Studies were considered eligible if they met the following inclusion criteria.

\section{Type of participants}

Adult patients (> 18 years old) undergoing surgery were included as participants. The patients were defined as high risk when they fulfilled at least one of the patient-related or surgery-related criteria. The patient-related criteria were age >60 years or American Society of Anesthesiologists (ASA) score $\geq 3$ due to any reason. The surgery-related criteria were high-risk surgeries defined by original studies and by European Society of Cardiology/ European Society of Anesthesiology (ESC/ESA) guidelines [12], including emergency surgery, cardiac surgery, major vascular surgery, major abdominal surgery, or surgeries with presumed blood loss $>20 \%$ of blood volume. 


\section{Type of intervention}

The intervention was defined as GDFT based on dynamic variables derived from cardiorespiratory interaction, including SVV, SPV, PPV and PVI. Variables not derived from cardiorespiratory interaction were considered as other optimization goals, such as $\mathrm{CO}, \mathrm{CI}$, and oxygen delivery.

\section{Type of comparison}

Comparison of the effects of GDFTdyn with those of standard fluid management was considered. Standard fluid management was defined as fluid management based on standard physiologic variables, such as heart rate, blood pressure, central venous pressure (CVP) or urine output.

\section{Type of outcome measures}

The primary outcomes were short-term mortality and overall morbidity. Short-term mortality was defined as 30-day or hospital mortality. Overall morbidity was defined as the proportion of patients with one or more postoperative complications. The secondary outcomes were serum lactate concentration at the end of surgery, organ-specific morbidity (neurological, cardiovascular, pulmonary, abdominal and renal complications), and length of stay in the ICU and in hospital. The organ-specific morbidity was defined as the proportion of patients with an organ-specific complication. These complications included neurological (stroke), cardiovascular (arrhythmia, myocardial infarction, heart failure/cardiovascular dysfunction), pulmonary (acute lung injury/acute respiratory distress syndrome (ALI/ARDS), pneumonia, pulmonary embolism), abdominal (gastrointestinal (GIT) bleeding, GIT obstruction) and renal (acute kidney injury (AKI), renal failure with dialysis) complications.

Studies were excluded if they did not report any of these clinical outcomes.

\section{Data extraction}

Data were independently extracted to a predesigned form by two authors (SHW and JTS). The following variables were collected: first author, year of publication, study design, patient demographics (age, sample size, ASA class, high or moderate risk), surgical variables (surgical procedure, duration of surgery, estimated blood loss), intraoperative fluid administration (GDFTdyn, other optimization goals, monitoring devices, fluid management), and outcomes (short-term mortality, overall morbidity, serum lactate concentration at the end of surgery, postoperative organ-specific complications, length of stay in ICU and hospital).

\section{Quality assessment}

The Cochrane Collaboration's tool for assessing risk of bias was applied. It focuses upon selection bias, performance bias, detection bias, attrition bias, and reporting bias.

\section{Statistical analysis}

We performed two separate analyses by pooling data from RCTs comparing GDFTdyn alone or GDFTdyn with other optimization goals with standard fluid therapy (analysis 1: GDFTdyn alone versus standard fluid therapy; analysis 2: GDFTdyn with other optimization goals versus standard fluid therapy, respectively). We divided the included studies into these two groups according to the combination of other optimization goals. Note that we did not take heart rate, blood pressure, CVP, and urine output into consideration of other optimization goals because normalization of them could not prevent the occurrence of occult tissue hypoxia [3, 4]. Sensitivity analysis was conducted after excluding studies with high risk of bias. Subgroup analyses were conducted according to the type of surgery (cardiac or non-cardiac), patient risk (high or moderate risk), fluid management (fluid with or without inotropes), and monitoring devices (minimally invasive or non-invasive).

Statistical analysis was performed using Review Manager 5.3 software (Cochrane Collaboration, Denmark). Dichotomous data outcomes were analyzed using Mantel-Haenszel random-effects model and results presented as odds ratios (OR) with $95 \%$ confidence intervals (CI). Continuous data outcomes were analyzed using inverse variance random-effects modeling and quoted as mean differences (MD) with $95 \%$ CIs. A statistically significant difference between groups was considered to be present if the pooled 95\% CI did not include 0 for respective MD or 1 for respective OR. Statistical heterogeneity was assessed by $I$-square test and considered to be significant if $I$-square was $>75 \%$.

\section{Results}

\section{Study selection and characteristics}

After removal of duplicates, a total of 794 studies remained: 81 studies were reviewed in full and 37 studies finally met the inclusion criteria. The process of literature searching, screening and selection is presented in Additional file 2. The 37 studies included a total of 2910 patients, 1456 in the GDFTdyn arm and 1454 in the standard fluid therapy arm [13-49]. Patients in 27 studies were defined as high risk due to patient-related or surgery-related reasons. Of all included studies, 20 studies were based in abdominal surgery, 5 in cardiovascular, 3 in neurological, 2 in head and neck, 2 in thoracic, 1 in orthopedic and 1 in urologic surgery. Analysis 1 included 11 studies and analysis 2 included 26 studies. SVV, PVV, SPV and PVI were measured as GDFTdyn endpoints. $\mathrm{CO}$ or $\mathrm{CI}$ was the common or even the only goal except for GDFTdyn endpoints in almost all studies included in analysis 2 . The characteristics of the included studies are summarized in Table 1 . 


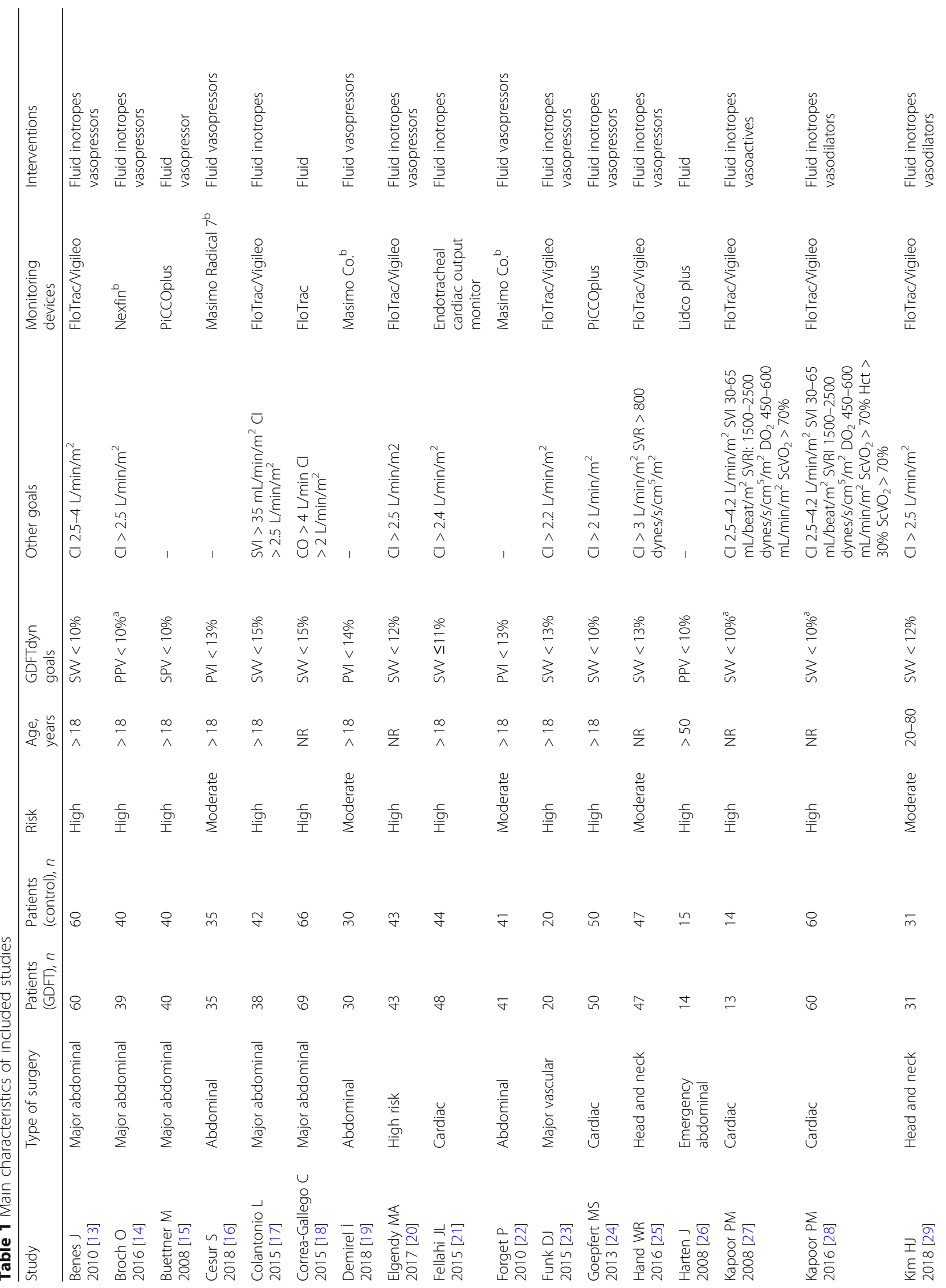




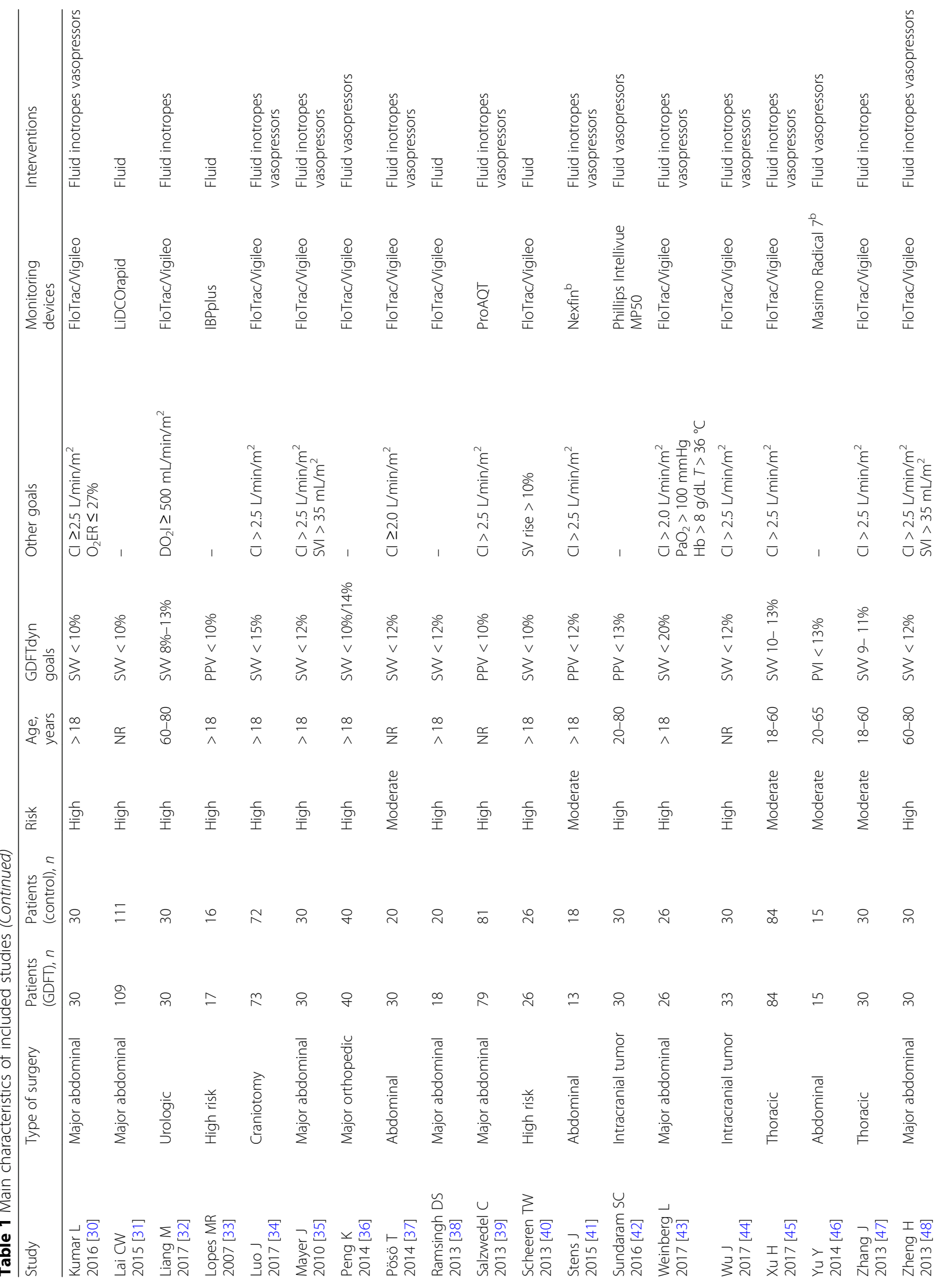




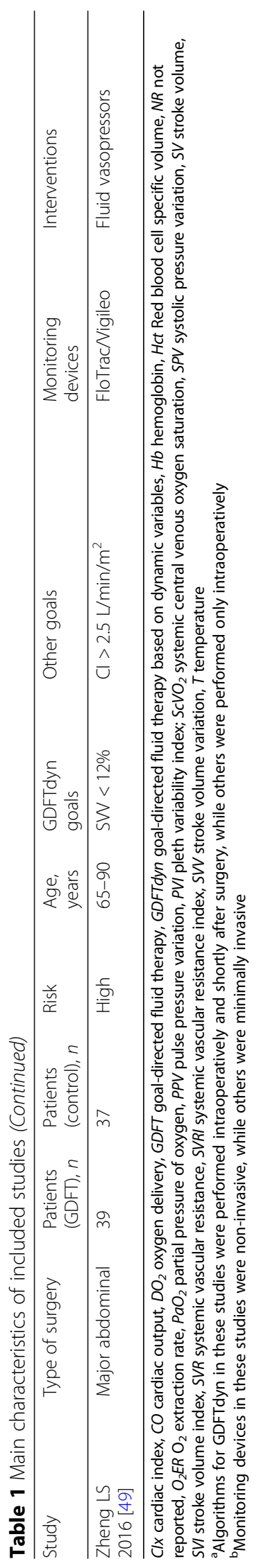




\section{Quality assessment}

Risk of bias was assessed by the Cochrane Collaboration's tool. The methodological quality of the included studies is summarized in Additional file 3. Random sequence generation was clearly reported in 30 of the included studies and allocation concealment in 22 studies: 17 of the studies clearly stated the blinding of participants, and 24 of the studies clearly reported blinding of the outcome assessment. Incomplete outcome data were not clearly reported in six studies. Selective reporting was found only in one study.

\section{Meta-analyses}

\section{Analysis 1: GDFTdyn alone versus standard fluid therapy}

Primary outcomes Six studies including 524 patients reported postoperative short-term mortality. The meta-analysis of these trials showed no significant difference between the patients managed with GDFTdyn alone and those with standard fluid therapy (OR $0.85,95 \%$ CI $(0.32$, 2.24), $P=0.74, \mathrm{I}^{2}=0 \%$ ) (Fig. 1). Sensitive analysis excluding studies with high risk of bias also showed no significant difference between two groups (Additional file 4). No significant difference was found between two groups among any subgroup analyses (Table 3).

Three studies including 282 patients reported postoperative overall morbidity. No significant difference was observed between GDFTdyn alone and standard fluid therapy group (OR $1.03,95 \% \mathrm{CI}(0.31,3.37), P=0.97$, $\mathrm{I}^{2}=67 \%$ ) (Fig. 2). Sensitive analysis excluding studies with high risk of bias also showed no significant difference between two groups (Additional file 5). No significant difference was found between two groups in any subgroup analyses (Table 3).

\section{Secondary outcomes}

Serum lactate concentration was significantly lower in patients managed with GDFTdyn alone (MD $-0.21 \mathrm{mmol} /$ L, 95\% CI $\left.(-0.39,-0.03), P=0.02, \mathrm{I}^{2}=82 \%\right)$ (Fig. 3). However, no significant difference was found between two groups in any organ-specific morbidity (Table 2), length of stay in ICU (MD $-0.26 \mathrm{~d}, 95 \%$ CI $(-2.00,1.47), P=0.77$, $\mathrm{I}^{2}=0 \%$ ) (Fig. 4) and hospital (MD 0.19d, 95\% CI (-1.11, 1.49), $\mathrm{P}=0.77, \mathrm{I}^{2}=41 \%$ ) (Fig. 5). The reduction in serum lactate concentration persisted in non-cardiac surgery, high-risk patients, fluid management without inotropes and minimally invasive monitoring device subgroups. No significant difference was found in length of stay in ICU and hospital among any subgroup analyses (Table 3 ).

\section{Analysis 2: GDFTdyn with other optimization goals versus standard fluid therapy Primary outcomes}

Postoperative short-term mortality was reported in 13 studies including 1100 patients. Compared with standard fluid therapy, a significant reduction in short-term mortality was observed in favor of GDFTdyn with other optimization goals (OR 0.45, 95\% CI $\left.(0.24,0.85), P=0.01, I^{2}=0 \%\right)$ (Fig. 1). Sensitivity analysis excluding studies with high risk of bias also showed significant reduction in short-term

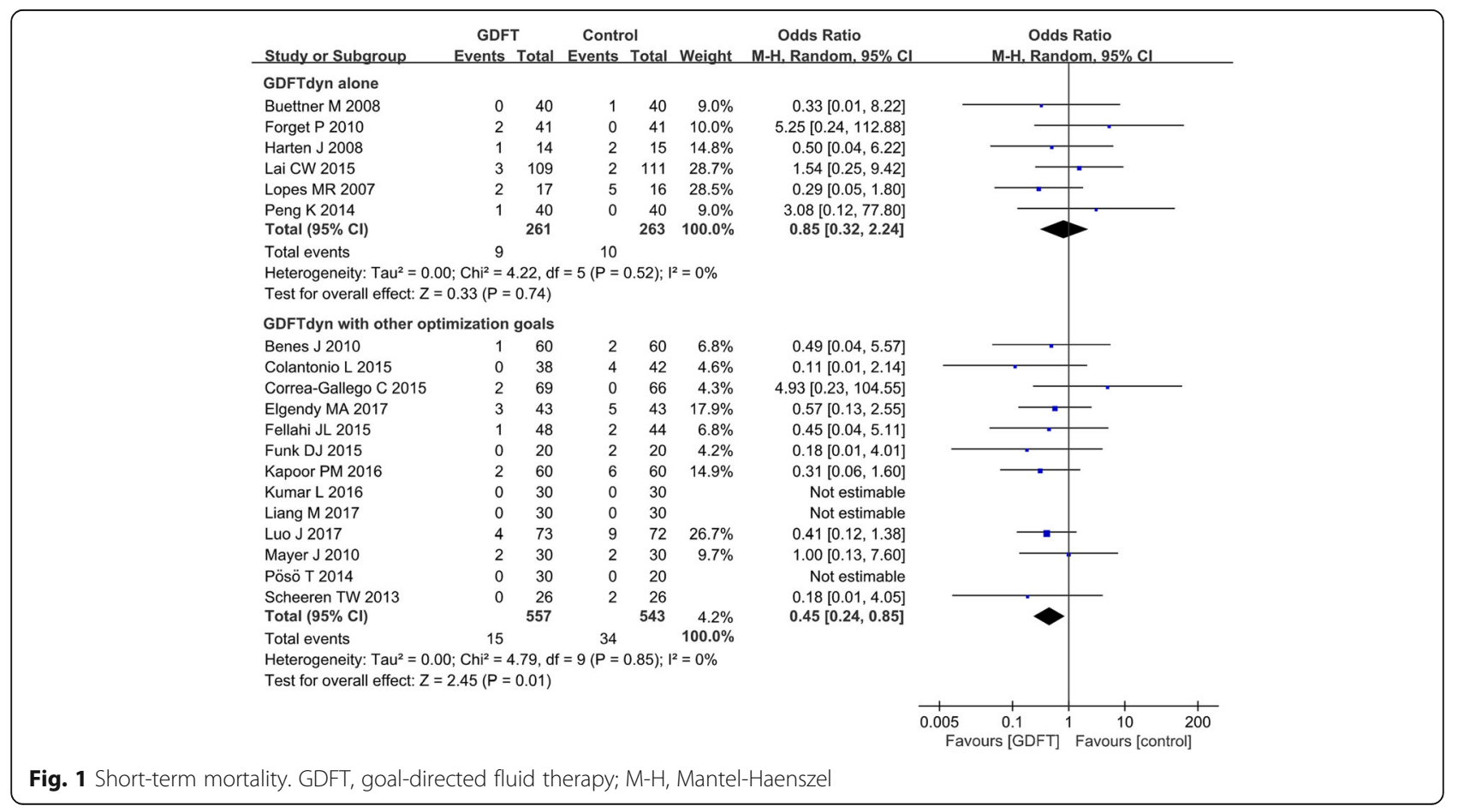




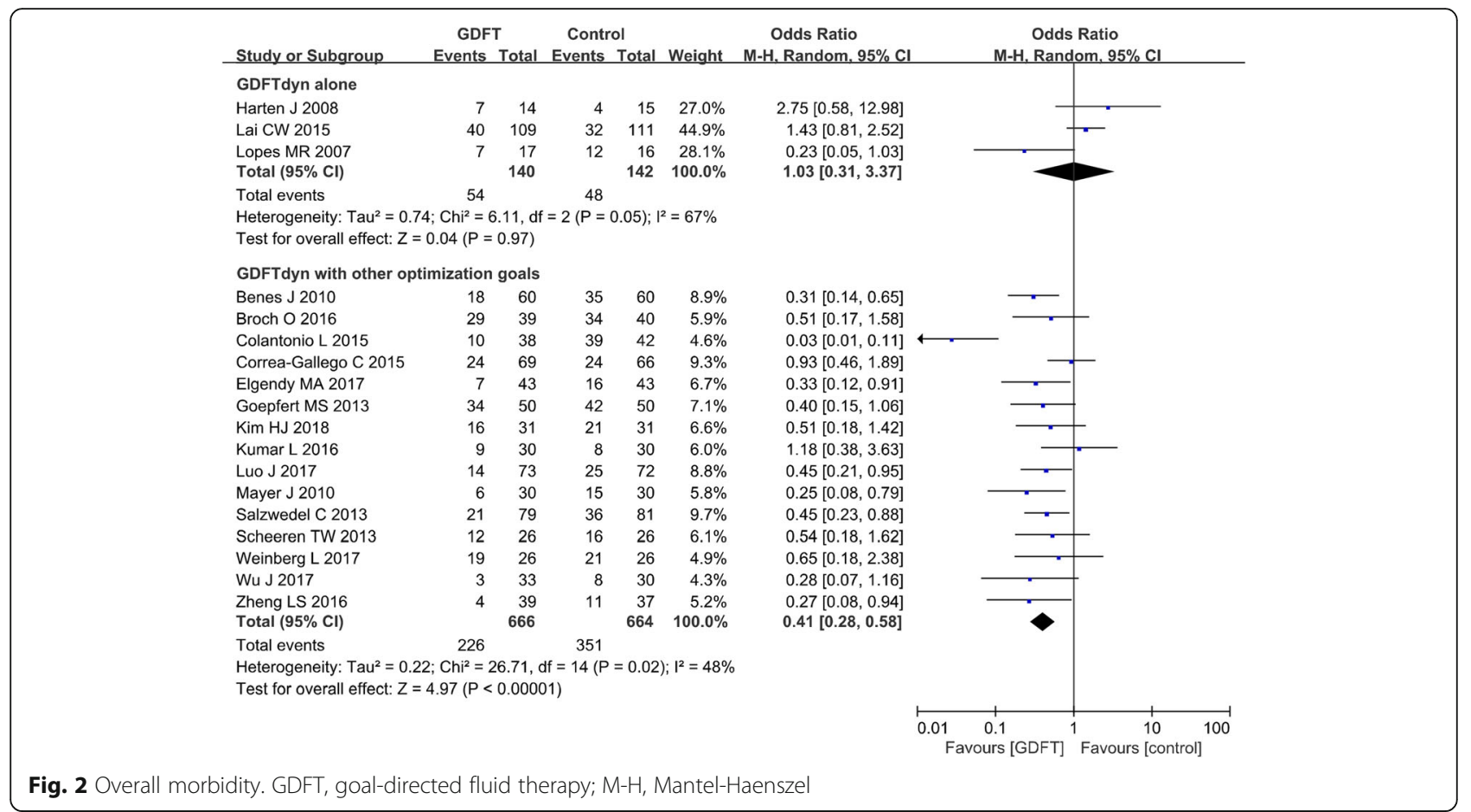

mortality by GDFTdyn with other optimization goals (Additional file 4). Subgroup analyses showed that the reduction in short-term mortality was associated with high-risk patients, the use of fluid and inotropes, and minimally invasive monitoring devices (Table 3).

Postoperative overall morbidity was reported in 15 studies with 1330 patients. Overall morbidity was significantly reduced in patients managed with GDFTdyn and other optimization goals when compared with those managed with standard care (OR 0.41, 95\% CI $(0.28,0.58), P<0.00001$, $I^{2}=48 \%$ ) (Fig. 2). Sensitivity analysis excluding studies with high risk of bias also showed significant reduction in overall morbidity by GDFTdyn with other optimization goals (Additional file 5). Also, subgroup analysis showed that the

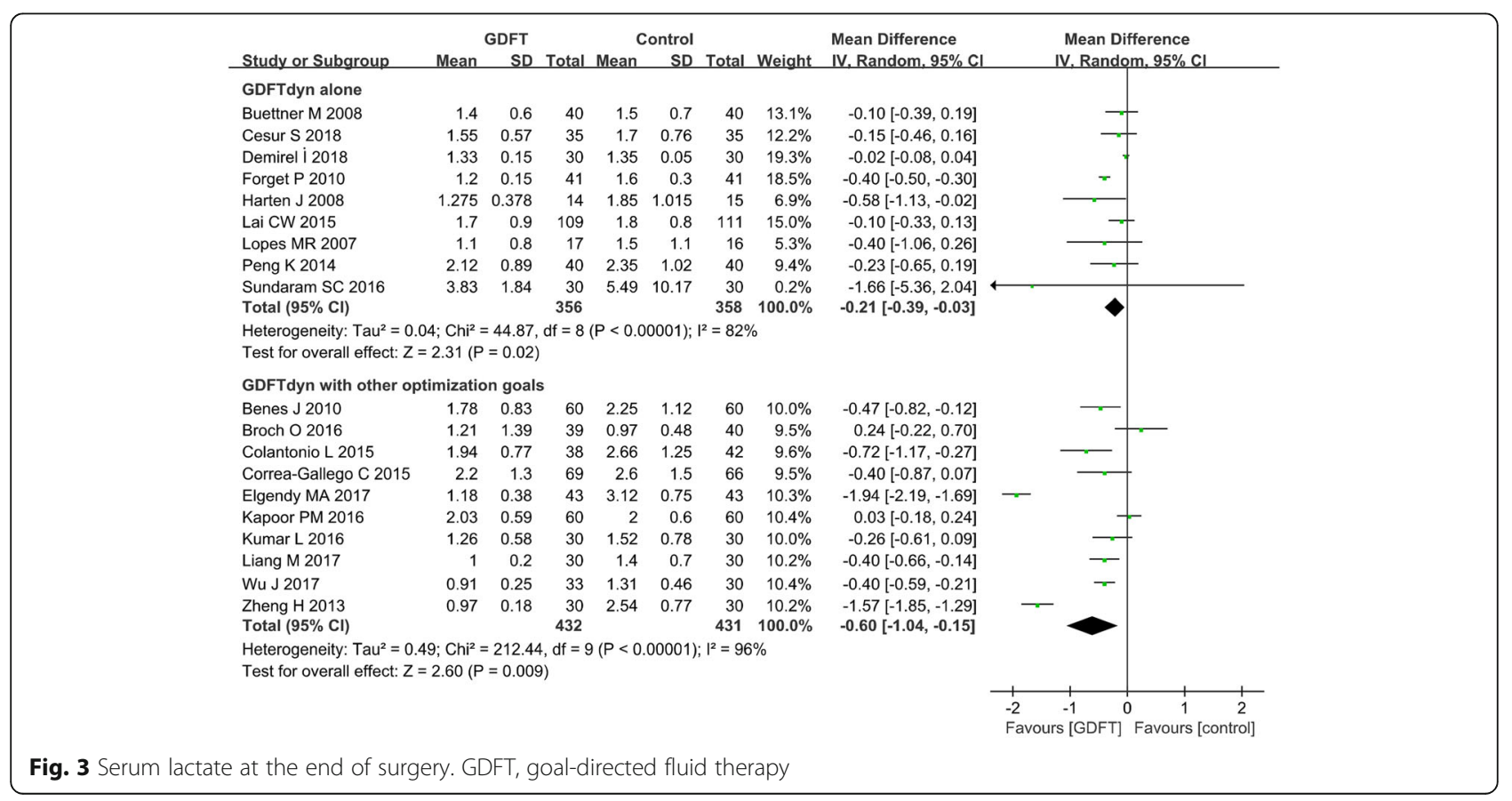


Deng et al. Critical Care $\quad$ (2018) 22:298

Page 9 of 17

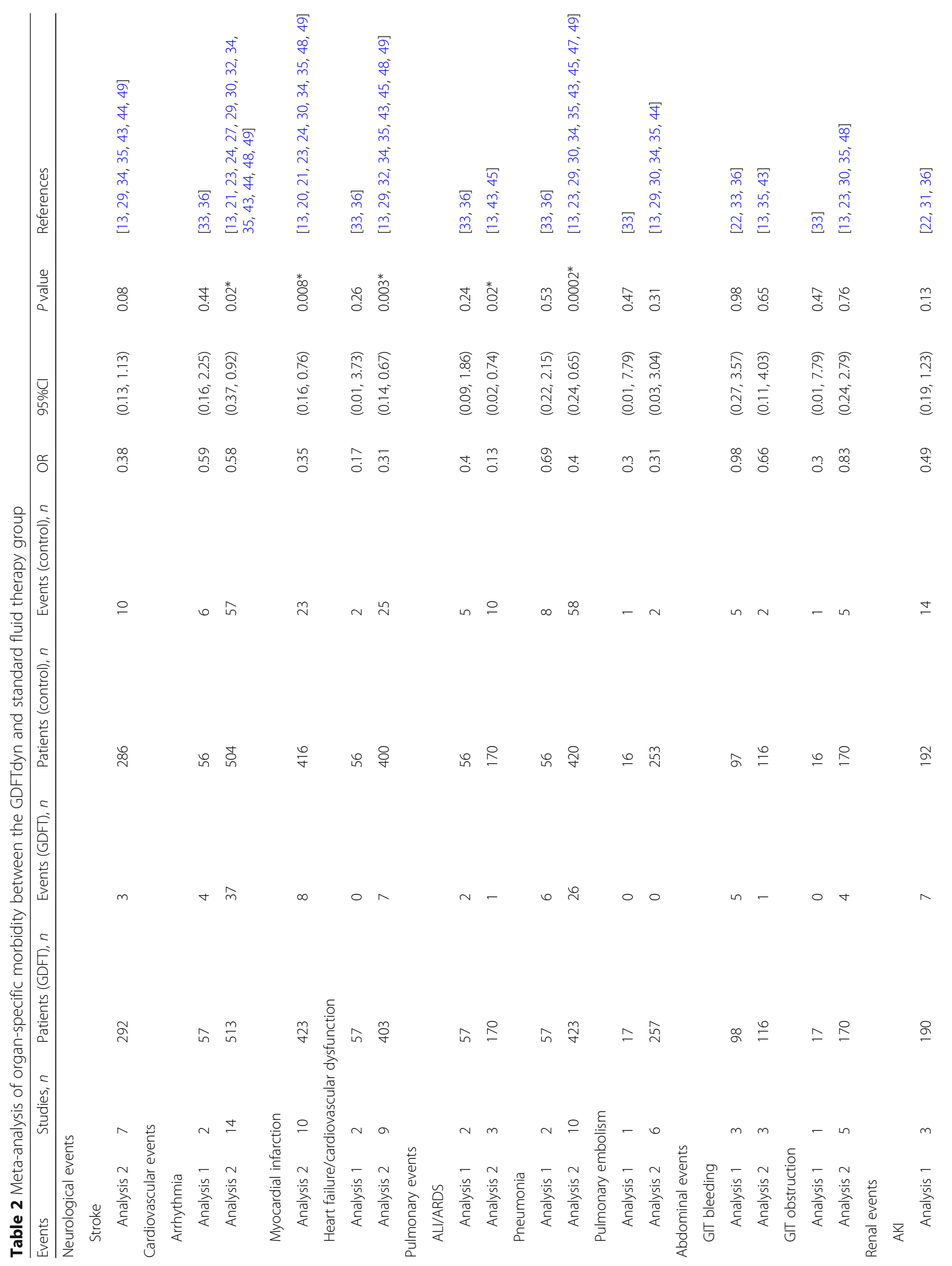




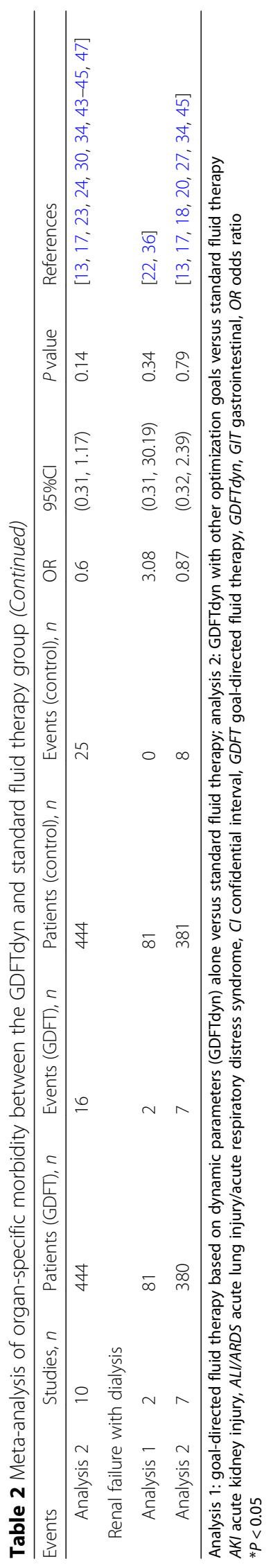




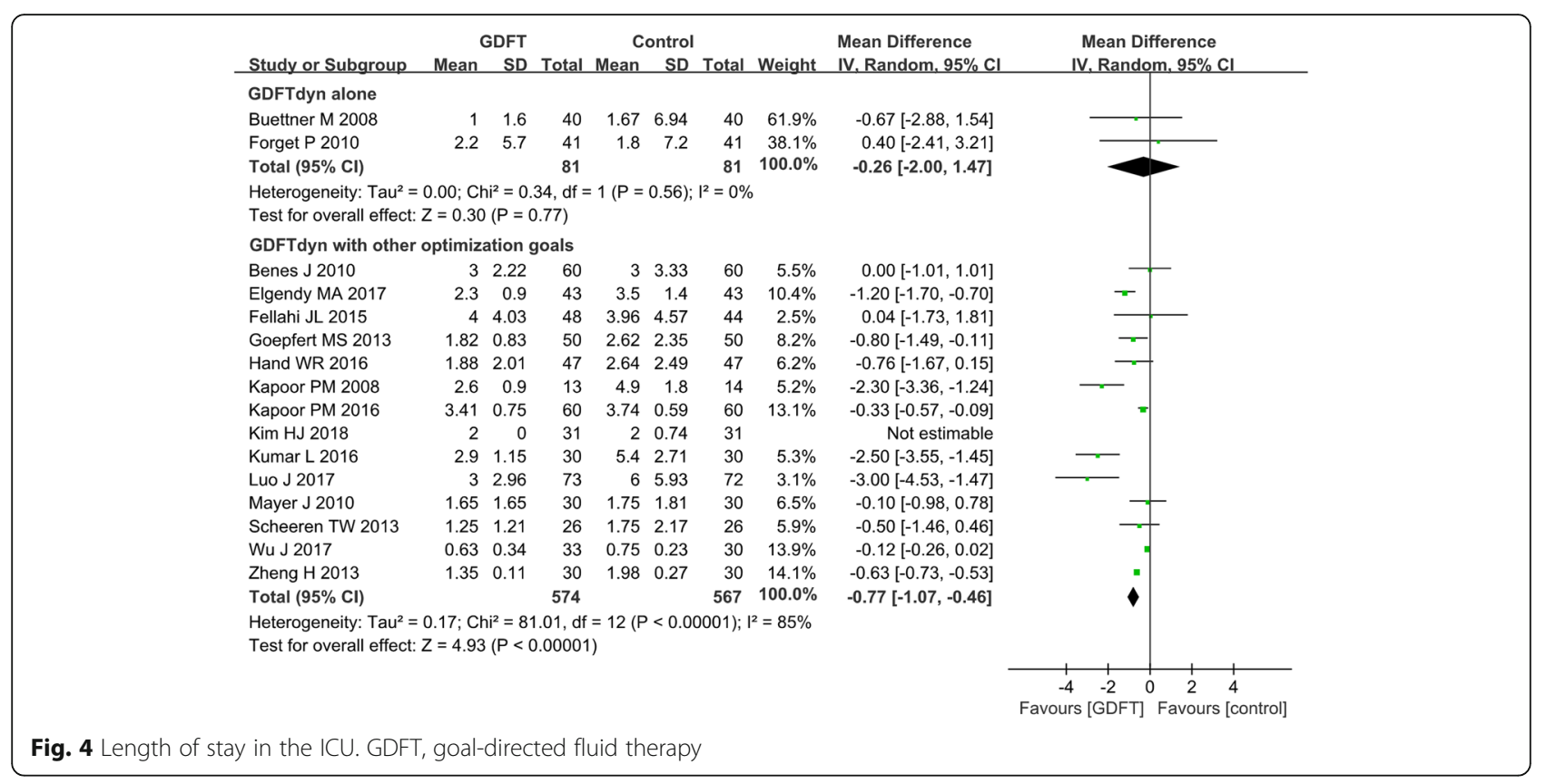

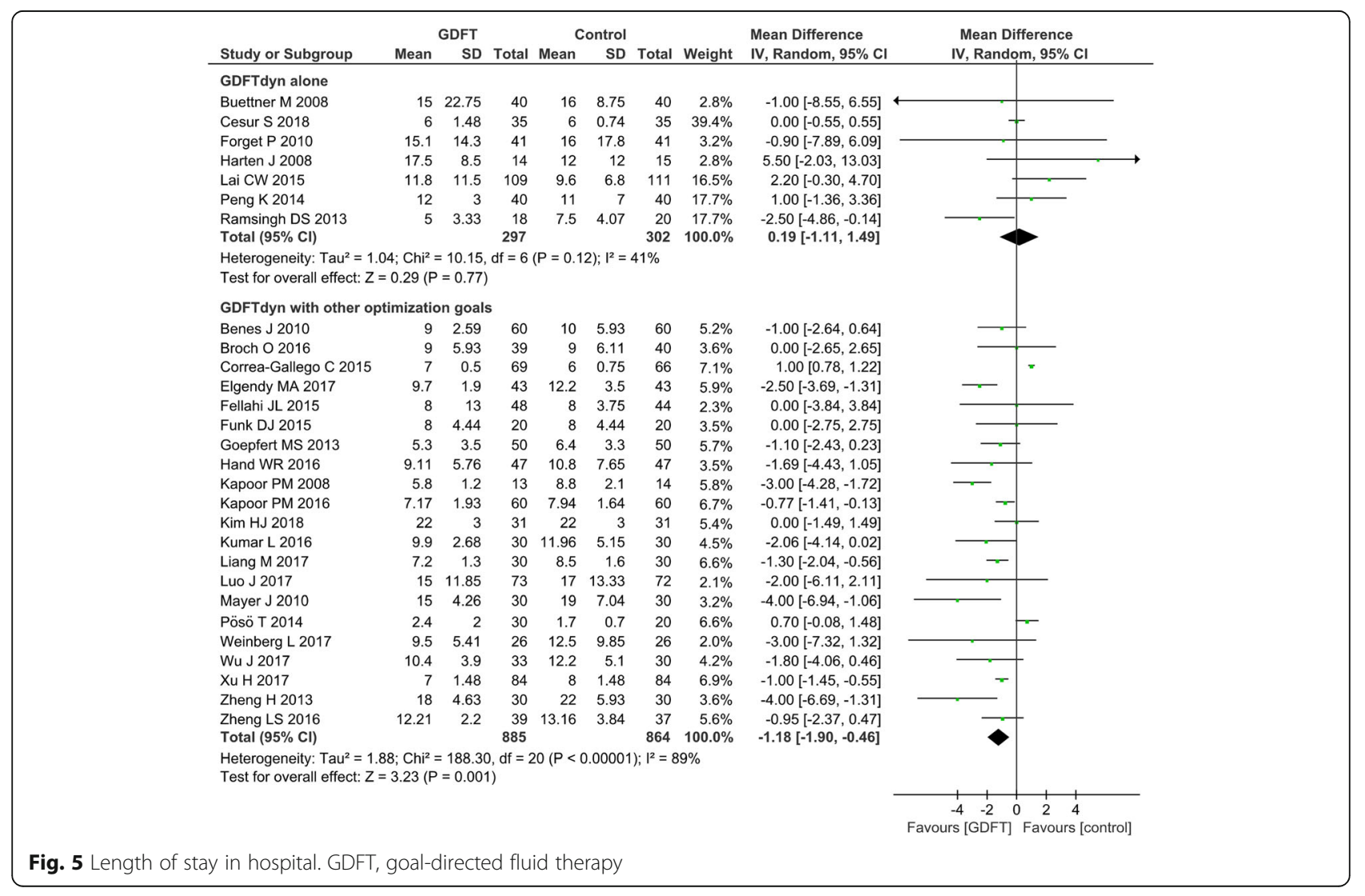


Table 3 Subgroup analyses of clinical outcomes between the GDFTdyn and standard fluid therapy group

\begin{tabular}{|c|c|c|c|c|c|c|c|c|}
\hline \multirow[t]{2}{*}{ Subgroups } & \multicolumn{4}{|l|}{ Analysis 1} & \multicolumn{4}{|l|}{ Analysis 2} \\
\hline & Studies, $n$ & $\mathrm{OR} / \mathrm{MD}$ & $95 \% \mathrm{Cl}$ & $P$ value & Studies, $n$ & OR/MD & $95 \% \mathrm{Cl}$ & $P$ value \\
\hline \multicolumn{9}{|l|}{ Short-term mortality } \\
\hline \multicolumn{9}{|l|}{ Surgery } \\
\hline Non-cardiac & 6 & 0.85 & $(0.32,2.24)$ & 0.74 & 11 & 0.49 & $(0.24,1.00)$ & 0.05 \\
\hline Cardiac & - & - & - & - & 2 & 0.35 & $(0.09,1.36)$ & 0.13 \\
\hline \multicolumn{9}{|l|}{ risk } \\
\hline High & 5 & 0.69 & $(0.25,1.93)$ & 0.48 & 12 & 0.45 & $(0.24,0.85)$ & $0.01^{*}$ \\
\hline Moderate & 1 & 5.25 & $(0.24,112.8)$ & 0.29 & - & - & - & - \\
\hline \multicolumn{9}{|l|}{ Fluid/inotropes } \\
\hline Fluid & 6 & 0.85 & $(0.32,2.24)$ & 0.74 & 2 & 0.96 & $(0.04,23.99)$ & 0.98 \\
\hline Fluid+inotropes & - & - & - & - & 11 & 0.42 & $(0.22,0.82)$ & $0.01^{*}$ \\
\hline \multicolumn{9}{|l|}{ Monitoring devices } \\
\hline Minimally invasive & 5 & 0.69 & $(0.25,1.93)$ & 0.48 & 13 & 0.45 & $(0.24,0.85)$ & $0.01^{*}$ \\
\hline Non-invasive & 1 & 5.25 & $(0.24,112.8)$ & 0.29 & - & - & - & - \\
\hline \multicolumn{9}{|l|}{ Overall morbidity } \\
\hline \multicolumn{9}{|l|}{ Surgery } \\
\hline Non-cardiac & 3 & 1.03 & $(0.31,3.37)$ & 0.97 & 14 & 0.4 & $(0.28,0.59)$ & $<0.00001^{*}$ \\
\hline Cardiac & - & - & - & - & 1 & 0.4 & $(0.15,1.06)$ & 0.07 \\
\hline \multicolumn{9}{|l|}{ risk } \\
\hline High & 3 & 1.03 & $(0.31,3.37)$ & 0.97 & 14 & 0.4 & $(0.27,0.58)$ & $<0.00001^{*}$ \\
\hline Moderate & - & - & - & - & 1 & 0.51 & $(0.18,1.42)$ & 0.2 \\
\hline \multicolumn{9}{|l|}{ Fluid/inotropes } \\
\hline Fluid & 3 & 1.03 & $(0.31,3.37)$ & 0.97 & 3 & 0.6 & $(0.30,1.20)$ & 0.15 \\
\hline Fluid+inotropes & - & - & - & - & 12 & 0.37 & $(0.25,0.55)$ & $<0.00001^{*}$ \\
\hline \multicolumn{9}{|l|}{ Monitoring devices } \\
\hline Minimally invasive & 3 & 1.03 & $(0.31,3.37)$ & 0.97 & 14 & 0.4 & $(0.27,0.58)$ & $<0.00001^{*}$ \\
\hline Non-invasive & - & - & - & - & 1 & 0.51 & $(0.17,1.58)$ & 0.24 \\
\hline \multicolumn{9}{|c|}{ Serum lactate concentration } \\
\hline \multicolumn{9}{|l|}{ Surgery } \\
\hline Non-cardiac & 9 & -0.21 & $(-0.39,-0.03)$ & $0.02^{*}$ & 9 & -0.67 & $(-1.14,-0.20)$ & $0.005^{*}$ \\
\hline Cardiac & - & - & - & - & 1 & 0.03 & $(-0.18,0.24)$ & 0.78 \\
\hline \multicolumn{9}{|l|}{ risk } \\
\hline High & 6 & -0.17 & $(-0.32,-0.02)$ & $0.03^{*}$ & 10 & -0.6 & $(-1.04,-0.15)$ & $0.009^{*}$ \\
\hline Moderate & 3 & -0.19 & $(-0.49,0.11)$ & 0.21 & - & - & - & - \\
\hline \multicolumn{9}{|l|}{ Fluid/inotropes } \\
\hline Fluid & 9 & -0.21 & $(-0.39,-0.03)$ & $0.02^{*}$ & 1 & -0.4 & $(-0.87,0.07)$ & 0.1 \\
\hline Fluid+inotropes & - & - & - & - & 9 & -0.62 & $(-1.10,-0.13)$ & $0.01^{*}$ \\
\hline \multicolumn{9}{|l|}{ Monitoring devices } \\
\hline Minimally invasive & 6 & -0.17 & $(-0.32,-0.02)$ & $0.03^{*}$ & 9 & -0.68 & $(-1.15,-0.22)$ & $0.004^{*}$ \\
\hline Non-invasive & 3 & -0.19 & $(-0.49,0.11)$ & 0.21 & 1 & 0.24 & $(-0.22,0.70)$ & 0.31 \\
\hline \multicolumn{9}{|l|}{ length of stay in ICU } \\
\hline \multicolumn{9}{|l|}{ Surgery } \\
\hline Non-cardiac & 2 & -0.26 & $(-2.00,1.47)$ & 0.77 & 10 & -0.77 & $(-1.15,-0.39)$ & $<0.0001^{*}$ \\
\hline Cardiac & - & - & - & - & 4 & -0.86 & $(-1.68,-0.04)$ & $0.04^{*}$ \\
\hline
\end{tabular}


Table 3 Subgroup analyses of clinical outcomes between the GDFTdyn and standard fluid therapy group (Continued)

\begin{tabular}{|c|c|c|c|c|c|c|c|c|}
\hline \multirow[t]{2}{*}{ Subgroups } & \multicolumn{4}{|l|}{ Analysis 1} & \multicolumn{4}{|l|}{ Analysis 2} \\
\hline & Studies, $n$ & OR/MD & $95 \% \mathrm{Cl}$ & $P$ value & Studies, $n$ & $\mathrm{OR} / \mathrm{MD}$ & $95 \% \mathrm{Cl}$ & $P$ value \\
\hline \multicolumn{9}{|l|}{ Risk } \\
\hline High & 2 & -0.26 & $(-2.00,1.47)$ & 0.77 & 12 & -0.77 & $(-1.09,-0.45)$ & $<0.00001^{*}$ \\
\hline Moderate & - & - & - & - & 2 & -0.76 & $(-1.67,0.15)$ & 0.1 \\
\hline \multicolumn{9}{|l|}{ Fluid/inotropes } \\
\hline Fluid & 2 & -0.26 & $(-2.00,1.47)$ & 0.77 & 1 & -0.5 & $(-1.46,0.46)$ & 0.3 \\
\hline Fluid+inotropes & - & - & - & - & 13 & -0.79 & $(-1.10,-0.47)$ & $<0.00001^{*}$ \\
\hline \multicolumn{9}{|l|}{ Monitoring devices } \\
\hline Minimally invasive & 1 & -0.67 & $(-2.88,1.54)$ & 0.55 & 14 & -0.77 & $(-1.07,-0.46)$ & $<0.00001^{*}$ \\
\hline Non-invasive & 1 & 0.4 & $(-2.41,3.21)$ & 0.78 & - & - & - & - \\
\hline \multicolumn{9}{|l|}{ length of stay in hospital } \\
\hline \multicolumn{9}{|l|}{ Surgery } \\
\hline Non-cardiac & 7 & 0.19 & $(-1.11,1.49)$ & 0.77 & 17 & -1.13 & $(-1.94,-0.32)$ & $0.006^{*}$ \\
\hline Cardiac & - & - & - & - & 4 & -1.42 & $(-2.63,-0.21)$ & $0.02^{*}$ \\
\hline \multicolumn{9}{|l|}{ Risk } \\
\hline High & 5 & 0.54 & $(-1.88,2.96)$ & 0.66 & 17 & -1.45 & $(-2.37,-0.52)$ & $0.002^{*}$ \\
\hline Moderate & 2 & -0.01 & $(-0.55,0.54)$ & 0.98 & 4 & -0.33 & $(-1.47,0.81)$ & 0.58 \\
\hline \multicolumn{9}{|l|}{ Fluid/inotropes } \\
\hline Fluid & 7 & 0.19 & $(-1.11,1.49)$ & 0.77 & 2 & 0.16 & $(-1.74,2.05)$ & 0.87 \\
\hline Fluid+inotropes & - & - & - & - & 19 & -1.28 & $(-1.82,-0.73)$ & $<0.00001^{*}$ \\
\hline \multicolumn{9}{|l|}{ Monitoring devices } \\
\hline Minimally invasive & 5 & 0.54 & $(-1.88,2.96)$ & 0.66 & 20 & -1.23 & $(-1.96,-0.49)$ & $0.001^{*}$ \\
\hline Non-invasive & 2 & -0.01 & $(-0.55,0.54)$ & 0.98 & 1 & 0 & $(-2.65,2.65)$ & 1 \\
\hline
\end{tabular}

Analysis 1: goal-directed fluid therapy based on dynamic parameters (GDFTdyn) alone versus standard fluid therapy; analysis 2: GDFTdyn with other optimization goals versus standard fluid therapy. Results for short-term mortality and overall morbidity are presented as odds ratio (OR) and $95 \%$ confidence interval (Cl). Results on serum lactate concentration and length of stay in the ICU and in hospital are presented as mean difference (MD) and $95 \% \mathrm{Cl}$ ICU intensive care unit

${ }^{*} P<0.05$

reduction of overall morbidity was associated with non-cardiac surgery, high-risk patients, the use of fluid and inotropes, and minimally invasive monitoring devices (Table 3).

\section{Secondary outcomes}

Compared with standard fluid therapy, serum lactate concentration (MD $-0.60 \mathrm{mmol} / \mathrm{L}, 95 \% \mathrm{CI}(-1.04,-0.15)$, $P=0.009, I^{2}=96 \%$ ) (Fig. 3), incidence of cardiovascular complications (arrhythmia, OR $0.58,95 \%$ CI $(0.37,0.92)$, $P=0.02, I^{2}=0 \%$; myocardial infarction, OR $0.35,95 \% \mathrm{CI}$ (0.16, 0.76), $P=0.008, I^{2}=0 \%$; heart failure/cardiovascular dysfunction, OR $0.31,95 \% \mathrm{CI}(0.14,0.67), P=0.003, I^{2}=$ $0 \%$ ), pulmonary complications (ALI/ARDS, OR 0.13, 95\% CI $(0.02,0.74), \mathrm{P}=0.02, I^{2}=0 \%$; pneumonia, OR $0.4,95 \%$ CI $(0.24,0.65), P=0.0002, I^{2}=0 \%$ ) (Table 2), and length of stay in the ICU (MD $-0.77 \mathrm{~d}, 95 \% \mathrm{CI}(-1.07,-0.46), P<$ $0.0001, I^{2}=85 \%$ ) (Fig. 4) and in hospital (MD -1.18 days, 95\% CI (-1.90, -0.46), $P=0.001, I^{2}=89 \%$ ) (Fig. 5) were significantly lower in patients managed with GDFTdyn with other optimization goals. The reduction in serum lactate concentration and length of stay in the ICU and in hospital persisted in high-risk patients, and in subgroups receiving fluid with inotropes and minimally invasive monitoring devices (Table 3).

\section{Discussion}

The current study demonstrated that GDFTdyn alone was not associated with improved clinical outcomes except for the reduction in serum lactate concentration. However, further analysis of studies evaluating GDFTdyn with other optimization goals (mainly $\mathrm{CO}$ or $\mathrm{CI}$ ) in their intervention arm revealed that the combination was associated with significant reduction in short-term mortality, overall morbidity, serum lactate concentration, cardiopulmonary complications, and length of stay in the ICU and in hospital.

Postoperative morbidity is as important as short-term mortality, for it might lead to loss of organ function and 
have an impact on long-term mortality [50]. Currently, evidence for the beneficial effects of GDFTdyn on mortality and morbidity has been inconsistent. Moreover, there is still no consensus on the most appropriate goals in GDFT strategies. Interestingly, our study revealed that optimization of fluid responsiveness by GDFTdyn alone was not associated with reduced mortality and morbidity. However, optimization of fluid responsiveness was found to be beneficial when it was in conjunction with other optimization goals (mainly $\mathrm{CO}$ or $\mathrm{CI}$ ) to optimize tissue and organ perfusion. Increasing cardiac contractility produces an increase in the slope of the Frank-Starling curve, such that patients on the flat section of the original curve move to a steeper section of the new curve [51]. Therefore, by reaching the goals of GDFTdyn and $\mathrm{CO} / \mathrm{CI}$ simultaneously, maximal stroke volume and adequate perfusion is achieved. Subgroup analyses also showed that the beneficial effects of GDFTdyn and other optimization goals persisted in patients using fluid and inotropes as the intervention. Another explanation for the improved clinical outcomes with the combination of GDFTdyn and $\mathrm{CO} / \mathrm{CI}$ goals might be the gray zone of GDFTdyn endpoints. The gray zone of these dynamic variables has been considered unable to reliably predict fluid responsiveness [52, 53]. Although we could not identify the exact proportion of patients with a gray zone value in the studies included in our analysis, reaching $\mathrm{CO} / \mathrm{CI}$ goals might prevent these patients from organ hypoperfusion. Our results contradicted a previous meta-analysis, which indicated a benefit of GDFTdyn compared to standard fluid therapy in reducing incidence of postoperative morbidity [5]. In their meta-analysis, 8 of 14 studies combined GDFTdyn endpoints with other optimization goals as interventions. Mixing studies on GDFTdyn alone with those on GDFTdyn with other optimization goals might lead to inaccurate or even erroneous conclusions.

High-risk patients undergoing surgery are thought to have higher oxygen demand and limited cardiopulmonary reserve. There is concern about GDFT-related cardiopulmonary complications in high-risk patients. Opposingly, we found that the improved clinical outcomes of GDFTdyn with $\mathrm{CO} / \mathrm{CI}$ goals persisted in high-risk patients. Especially, in the analysis of organ-specific morbidity, cardiopulmonary complications were significantly reduced by the combined goals. Another meta-analysis on high-risk surgery also showed the use of fluid and inotropes reduced the incidence of cardiac arrhythmia without increasing the incidence of acute pulmonary edema [8]. It seems that maximizing stroke volume and oxygen delivery is beneficial especially for high-risk patients, which might be attributed to improved tissue perfusion and cardiac performance.

Serum lactate concentration could serve as a sensitive biochemical variable of oxygen debt. The association between decreased serum lactate and a reduction in postoperative complications was found in previous studies [54]. In the current study, significant reduction in serum lactate and postoperative morbidity were also observed in the group with GDFTdyn and other optimization goals. However, in the GDFTdyn-alone group, serum lactate was lowered but reduction in postoperative morbidity was not observed. The reduction in serum lactate by GDFTdyn alone $(-0.21 \mathrm{mmol} / \mathrm{L})$ was much less than that by GDFTdyn with other optimization goals $(-0.60 \mathrm{mmol} / \mathrm{L})$. It might imply that GDFTdyn alone was less effective in correcting tissue hypoperfusion without other optimization goals.

Length of stay in the ICU and in hospital were also shorter in patients managed with GDFTdyn with other optimization goals but not in those managed with GDFTdyn alone, which was similar to the results for postoperative morbidity. It is possible that the significant reduction in length of stay mostly is attributed to the lower incidence of postoperative complications. The heterogeneity of length of stay in the ICU and in hospital in the group with GDFTdyn and other optimization goals was greater than $75 \%$. It might be attributed to the enormous change in the protocols and discharge criteria in the ICU and in hospital in recent years. Additionally, different units of measurement (days or hours) of length of stay in the ICU reported in different studies might also contribute to the heterogeneity.

Since the meta-analysis has several notable limitations, the results should be interpreted with caution. The main limitation was the clinical heterogeneity among different populations, surgical procedures, and monitoring devices. We tried to address the issue by the following measures. First, we divided the interventions into two groups and conducted two separate analyses (GDFTdyn alone versus standard fluid therapy and GDFTdyn with other optimization goals versus standard fluid therapy). Second, we conducted subgroup analyses according to the type of surgery (cardiac and non-cardiac), patient risk (high or moderate), fluid management (fluid with or without inotropes), and monitoring devices (minimally invasive or non-invasive). Finally, we used a random effect model to guarantee the robustness of the results and conclusions. Another limitation was failing to demonstrate a relationship between the year of publication of the included studies and the treatment effect. The included studies in the current meta-analysis spanned a long period of time. During this period, goal-directed fluid therapy has evolved rapidly and changed drastically. Also, fluid management in the postoperative period also has an important impact on clinical outcomes. However, postoperative fluid therapy regimes were not stated clearly in the included studies, making it difficult to evaluate the effects of them on perioperative outcomes. 


\section{Conclusions}

Based on the available data, we conclude that optimizing fluid responsiveness by GDFTdyn alone is not sufficient to improve clinical outcomes among patients undergoing surgery. However, the combination of GDFTdyn and other optimization goals to improve tissue and organ perfusion is associated with improved clinical outcomes. Patients managed with the combination of GDFTdyn and CO/CI goals might derive most benefit. High quality evidences with adequate statistical power and rigorous methodology are urgently needed to verify the beneficial effects of GDFT combined goals on clinical outcomes of patients undergoing surgery. Further researches are required to determine the most beneficial protocol and timing of GDFT strategies among different type of surgery (cardiac and non-cardiac) and different surgical populations (high or moderate risk).

\section{Additional files}

Additional file 1: PRISMA checklists. (DOCX $18 \mathrm{~kb}$ )

Additional file 2: Flow chart of literature searching, reviewing and selection. (TIF $512 \mathrm{~kb}$ )

Additional file $\mathbf{3}$ Risk of bias summary presenting judgments for each risk of bias item for each included study. (TIF 898 kb)

Additional file 4: Forest plot for short-term mortality among studies with low or moderate risk of bias. (TIF $869 \mathrm{~kb}$ )

Additional file 5: Forest plot for overall morbidity among studies with low or moderate risk of bias. (TIF 809 kb)

\section{Abbreviations}

AKl: Acute kidney injury; ALI/ARDS: Acute lung injury/acute respiratory distress syndrome; Cl: Confidence interval; Clx: Cardiac index; CO: Cardiac output; CVP: Central venous pressure; DO 2 : Oxygen delivery; GDFT: Goal-directed fluid therapy; GDFTdyn: Goal-directed fluid therapy based on dynamic variables; GIT: Gastrointestinal; Hb: Hemoglobin; Hct: Red blood cell specific volume; ICU: Intensive care unit; MD: Mean difference; NR: Not referred; $\mathrm{O}_{2} E R: \mathrm{O}_{2}$ extraction rate; OR: Odds ratio; $\mathrm{PaO}_{2}$ : Partial pressure of oxygen; PPV: Pulse pressure variation; PRISMA: Preferred Reporting Items for Systematic Reviews and Meta-Analyses; PVI: Pleth variability index; RCT: Randomized controlled trials; SAP: Systolic arterial pressure; $\mathrm{SCVO}_{2}$ : Systemic central venous oxygen saturation; SPV: Systolic pressure variation; SV: Stroke volume index; SVR: Systemic vascular resistance; SVRI: Systemic vascular resistance index; SW: Stroke volume variation; T: Temperature

\section{Acknowledgements}

We thank the authors and participants of the included studies for their important contributions.

\section{Funding}

None.

\section{Availability of data and materials}

The datasets generated and analyzed during the current study are available in the PubMed (https://www.ncbi.nlm.nih.gov/pubmed/), ClinicalTrials.gov (https:// www.clinicaltrials.gov/), EMBASE (https://www.elsevier.com/solutions/embasebiomedical-research) and the Cochrane Library (http://www.cochranelibrary.com/).

\section{Authors' contributions}

QWD and MX: study conception and design, acquisition, analysis, and interpretation of data, drafting the article, and revising it critically for important intellectual content; WCT and BCZ: study design, acquisition, analysis, and interpretation of data, and drafting the article; SHW and JTS: acquisition, analysis, and interpretation of data. All authors read and approved the final manuscript, and agreed to be accountable for all aspects of the work.
Ethics approval and consent to participate

Not applicable.

\section{Consent for publication}

Not applicable.

\section{Competing interests}

The authors declare that they have no competing interests.

\section{Publisher's Note}

Springer Nature remains neutral with regard to jurisdictional claims in published maps and institutional affiliations.

\section{Author details}

${ }^{1}$ Department of Anesthesiology, the First Affiliated Hospital, Sun Yat-sen University, No.58, Zhongshan 2nd Road, Guangzhou 510080, China. ${ }^{2}$ Department of Endoscopy, Sun Yat-sen University Cancer Center, No. 651, Dongfeng East Road, Guangzhou 510060, China. ${ }^{3}$ Department of Anesthesiology, Nanfang Hospital, Southern Medical University, No. 1838, Guangzhou Avenue North, Guangzhou 510515, China.

Received: 22 June 2018 Accepted: 29 October 2018

Published online: 14 November 2018

References

1. Khuri SF, Henderson WG, DePalma RG, Mosca C, Healey NA, Kumbhani DJ. Determinants of long-term survival after major surgery and the adverse effect of postoperative complications. Ann Surg. 2005;242(3):326-41.

2. Shin CH, Long DR, McLean D, Grabitz SD, Ladha K, Timm FP, et al. Effects of intraoperative fluid management on postoperative outcomes: a hospital registry study. Ann Surg. 2018;267(6):1084-92.

3. Marik PE, Cavallazzi R. Does the central venous pressure predict fluid responsiveness? An updated meta-analysis and a plea for some common sense. Crit Care Med. 2013;41(7):1774-81.

4. Meregalli A, Oliveira RP, Friedman G. Occult hypoperfusion is associated with increased mortality in hemodynamically stable, high-risk, surgical patients. Crit Care. 2004;8(2):R60-5.

5. Benes J, Giglio M, Brienza N, Michard F. The effects of goal-directed fluid therapy based on dynamic parameters on post-surgical outcome: a metaanalysis of randomized controlled trials. Crit Care. 2014;18(5):584.

6. Bednarczyk JM, Fridfinnson JA, Kumar A, Blanchard L, Rabbani R, Bell D, et al. Incorporating dynamic assessment of fluid responsiveness into goaldirected therapy: a systematic review and meta-analysis. Crit Care Med. 2017;45(9):1538-45.

7. Chong MA, Wang Y, Berbenetz NM, McConachie I. Does goal-directed haemodynamic and fluid therapy improve peri-operative outcomes? A systematic review and meta-analysis. Eur J Anaesthesiol. 2018;35(7):469-83.

8. Arulkumaran N, Corredor C, Hamilton MA, Ball J, Grounds RM, Rhodes A, et al. Cardiac complications associated with goal-directed therapy in high-risk surgical patients: a meta-analysis. Br J Anaesth. 2014;112(4):648-59.

9. Kaufmann T, Clement RP, Scheeren TWL, Saugel B, Keus F, van der Horst ICC. Perioperative goal-directed therapy: a systematic review without metaanalysis. Acta Anaesthesiol Scand. 2018. https://doi.org/10.1111/aas.13212.

10. Higgins J, Green S. Cochrane handbook for systematic reviews of interventions, version 5.1.0 [updated March 2011]. Cochrane Collaboration, 2008. Cochrane Collaboration website. www.cochranehandbook.org. Accessed 9 May 2018.

11. Moher D, Liberati A, Tetzlaff J, Altman DG. Preferred reporting items for systematic reviews and meta-analyses: the PRISMA statement. BMJ. 2009; 339:b2535.

12. Kristensen SD, Knuuti J, Saraste A, Anker S, Botker HE, Hert SD, et al. 2014 ESC/ESA guidelines on non-cardiac surgery: cardiovascular assessment and management: The Joint Task Force on non-cardiac surgery: cardiovascular assessment and management of the European Society of Cardiology (ESC) and the European Society of Anaesthesiology (ESA). Eur Heart J. 2014;35(35): 2383-431.

13. Benes J, Chytra I, Altmann P, Hluchy M, Kasal E, Svitak R, et al. Intraoperative fluid optimization using stroke volume variation in high risk surgical patients: results of prospective randomized study. Crit Care. 2010;14(3):R118. 
14. Broch O, Carstens A, Gruenewald M, Nischelsky E, Vellmer L, Bein B, et al. Non-invasive hemodynamic optimization in major abdominal surgery: a feasibility study. Minerva Anestesiol. 2016;82(11):1158-69.

15. Buettner M, Schummer W, Huettemann E, Schenke S, van Hout N, Sakka SG. Influence of systolic-pressure-variation-guided intraoperative fluid management on organ function and oxygen transport. Br J Anaesth. 2008;101(2):194-9.

16. Cesur S, Cardakozu T, Kus A, Turkyilmaz N, Yavuz O. Comparison of conventional fluid management with PVI-based goal-directed fluid management in elective colorectal surgery. J Clin Monit Comput. 2018. https://doi.org/10.1007/s10877-018-0163-y.

17. Colantonio L, Claroni C, Fabrizi L, Marcelli ME, Sofra M, Giannarelli D, et al. A randomized trial of goal directed vs. standard fluid therapy in cytoreductive surgery with hyperthermic intraperitoneal chemotherapy. J Gastrointest Surg. 2015;19(4):722-9.

18. Correa-Gallego C, Tan KS, Arslan-Carlon V, Gonen M, Denis SC, LangdonEmbry L, et al. Goal-directed fluid therapy using stroke volume variation for resuscitation after low central venous pressure-assisted liver resection: a randomized clinical trial. J Am Coll Surg. 2015;221(2):591-601.

19. Demirel I, Bolat E, Altun AY, Ozdemir M, Bestas A. Efficacy of goal-directed fluid therapy via pleth variability index during laparoscopic Roux-en-Y gastric bypass surgery in morbidly obese patients. Obes Surg. 2018;28(2):358-63.

20. Elgendy MA, Kassim DY, Esmat IM. Outcome of intraoperative goal-directed therapy using Vigileo/FloTrac in high-risk patients scheduled for major abdominal surgeries: a prospective randomized trial. Egyptian J Anaesth. 2017;33:263-9.

21. Fellahi $J$, Brossier D, Dechanet F, Fischer MO, Saplacan V, Gerard $J$, et al. Early goal-directed therapy based on endotracheal bioimpedance cardiography: a prospective, randomized controlled study in coronary surgery. J Clin Monit Comput. 2015;29(3):351-8.

22. Forget $P$, Lois $F$, de Kock M. Goal-directed fluid management based on the pulse oximeter-derived pleth variability index reduces lactate levels and improves fluid management. Anesth Analg. 2010;111(4):910-4.

23. Funk DJ, HayGlass KT, Koulack J, Harding G, Boyd A, Brinkman R. A randomized controlled trial on the effects of goal-directed therapy on the inflammatory response open abdominal aortic aneurysm repair. Crit Care. 2015;19:247.

24. Goepfert MS, Richter HP, Zu Eulenburg C, Gruetzmacher J, Rafflenbeul E, Roeher K, et al. Individually optimized hemodynamic therapy reduces complications and length of stay in the intensive care unit: a prospective, randomized controlled trial. Anesthesiology. 2013;119(4):824-36.

25. Hand WR, Stoll WD, McEvoy MD, MCSwain JR, Sealy CD, Skoner JM, et al. Intraoperative goal-directed hemodynamic management in free tissue transfer for head and neck cancer. Head Neck. 2016;38(Suppl 1):E1974-80.

26. Harten J, Crozier JE, McCreath B, Hay A, McMillan DC, McArdle CS, et al. Effect of intraoperative fluid optimisation on renal function in patients undergoing emergency abdominal surgery: a randomised controlled pilot study (ISRCTN 11799696). Int J Surg. 2008;6(3):197-204.

27. Kapoor PM, Kakani M, Chowdhury U, Choudhury M, Lakshmy KU. Early goaldirected therapy in moderate to high-risk cardiac surgery patients. Ann Card Anaesth. 2008;11(1):27-34

28. Kapoor PM, Magoon R, Rawat R, Mehta Y. Perioperative utility of goaldirected therapy in high-risk cardiac patients undergoing coronary artery bypass grafting: "a clinical outcome and biomarker-based study". Ann Card Anaesth. 2016;19(4):638-82.

29. Kim HJ, Kim EJ, Lee HJ, Min JY, Kim TW, Choi EC, et al. Effect of goaldirected haemodynamic therapy in free flap reconstruction for head and neck cancer. Acta Anaesthesiol Scand. 2018;62(7):903-14.

30. Kumar L, Rajan S, Baalachandran R. Outcomes associated with stroke volume variation versus central venous pressure guided fluid replacements during major abdominal surgery. J Anaesthesiol Clin Pharmacol. 2016;32(2):182-6.

31. Lai CW, Starkie T, Creanor S, Struthers RA, Portch D, Erasmus PD, et al. Randomized controlled trial of stroke volume optimization during elective major abdominal surgery in patients stratified by aerobic fitness. $\mathrm{Br} J$ Anaesth. 2015;115(4):578-89.

32. Liang M, Li Y, Lin L, Lin X, Wu X, Gao Y, et al. Effect of goal-directed fluid therapy on the prognosis of elderly patients with hypertension receiving plasma kinetic energy transurethral resection of prostate. Int J Clin Exp Med. 2017;10(1):1290-6.

33. Lopes MR, Oliveira MA, Pereira VO, Lemos IP, Auler JO Jr, Michard F. Goaldirected fluid management based on pulse pressure variation monitoring during high-risk surgery: a pilot randomized controlled trial. Crit Care. 2007; 11(5):R100.
34. Luo J, Xue J, Liu J, Liu B, Liu L, Chen G. Goal-directed fluid restriction during brain surgery: a prospective randomized controlled trial. Ann Intensive Care. 2017;7(1):16.

35. Mayer J, Boldt J, Mengistu AM, Rohm KD, Suttner S. Goal-directed intraoperative therapy based on autocalibrated arterial pressure waveform analysis reduces hospital stay in high-risk surgical patients: a randomized, controlled trial. Crit Care. 2010;14(1):R18.

36. Peng K, Li J, Cheng H, Ji FH. Goal-directed fluid therapy based on stroke volume variations improves fluid management and gastrointestinal perfusion in patients undergoing major orthopedic surgery. Med Princ Pract. 2014;23(5):413-20.

37. Poso $T$, Winso $O$, Aroch $R$, Kesek D. Perioperative fluid guidance with transthoracic echocardiography and pulse-contour device in morbidly obese patients. Obes Surg. 2014;24(12):2117-25.

38. Ramsingh DS, Sanghvi C, Gamboa J, Cannesson M, Applegate RL 2nd. Outcome impact of goal directed fluid therapy during high risk abdominal surgery in low to moderate risk patients: a randomized controlled trial. Clin Monit Comput. 2013;27(3):249-57.

39. Salzwedel C, Puig J, Carstens A, Bein B, Molnar Z, Kiss K, et al. Perioperative goal-directed hemodynamic therapy based on radial arterial pulse pressure variation and continuous cardiac index trending reduces postoperative complications after major abdominal surgery: a multi-center, prospective, randomized study. Crit Care. 2013;17(5):R191.

40. Scheeren TW, Wiesenack C, Gerlach H, Marx G. Goal-directed intraoperative fluid therapy guided by stroke volume and its variation in high-risk surgical patients: a prospective randomized multicentre study. J Clin Monit Comput. 2013;27(3):225-33.

41. Stens J, de Wolf SP, van der Zwan RJ, Koning NJ, Dekker NA, Hering JP, et al. Microcirculatory perfusion during different perioperative hemodynamic strategies. Microcirculation. 2015;22(4):267-75.

42. Sundaram SC, Salins SR, Kumar AN, Korula G. Intra-operative fluid management in adult neurosurgical patients undergoing intracranial tumour surgery: randomised control trial comparing pulse pressure variance (PPV) and central venous pressure (CVP). J Clin Diagn Res. 2016;10(5):UC01-5.

43. Weinberg L, lanno D, Churilov L, Chao I, Scurrah N, Rachbuch C, et al. Restrictive intraoperative fluid optimisation algorithm improves outcomes in patients undergoing pancreaticoduodenectomy: a prospective multicentre randomized controlled trial. PLoS One. 2017;12(9):e0183313.

44. Wu J, Ma Y, Wang T, Xu G, Fan L, Zhang Y. Goal-directed fluid management based on the auto-calibrated arterial pressure-derived stroke volume variation in patients undergoing supratentorial neoplasms surgery. Int J Clin Exp Med. 2017;10(2):3106-14

45. Xu H, Shu SH, Wang D, Chai XQ, Xie YH, Zhou WD. Goal-directed fluid restriction using stroke volume variation and cardiac index during one-lung ventilation: a randomized controlled trial. J Thorac Dis. 2017:9(9):2992-3004.

46. Yu Y, Dong J, Xu Z, Shen H, Zheng J. Pleth variability index-directed fluid management in abdominal surgery under combined general and epidural anesthesia. J Clin Monit Comput. 2015;29(1):47-52.

47. Zhang J, Chen CQ, Lei XZ, Feng ZY, Zhu SM. Goal-directed fluid optimization based on stroke volume variation and cardiac index during one-lung ventilation in patients undergoing thoracoscopy lobectomy operations: a pilot study. Clinics (Sao Paulo). 2013;68(7):1065-70.

48. Zheng H, Guo H, Ye JR, Chen L, Ma HP. Goal-directed fluid therapy in gastrointestinal surgery in older coronary heart disease patients: randomized trial. World J Surg. 2013;37(12):2820-9.

49. Zheng LS, Gu EW, Peng XH, Zhang L, Cao YY. Effect of goal-directed haemodynamic management on the postoperative outcome in elderly patients with fragile cardiac function undergoing abdominal surgery. Zhonghua Yi Xue Za Zhi. 2016;96(43):3464-9.

50. Jammer I, Wickboldt N, Sander M, Smith A, Schultz MJ, Pelosi P, et al. Standards for definitions and use of outcome measures for clinical effectiveness research in perioperative medicine: European Perioperative Clinical Outcome (EPCO) definitions: a statement from the ESA-ESICM joint taskforce on perioperative outcome measures. Eur J Anaesthesiol. 2015; 32(2):88-105.

51. Michard F, Lopes MR, Auler JO Jr. Pulse pressure variation: beyond the fluid management of patients with shock. Crit Care. 2007;11(3):131.

52. Cannesson M, Le Manach Y, Hofer CK, Goarin JP, Lehot JJ, Vallet $B$, et al. Assessing the diagnostic accuracy of pulse pressure variations for the prediction of fluid responsiveness: a "gray zone" approach. Anesthesiology. 2011;115(2):231-41. 
53. Biais M, Ehrmann S, Mari A, Conte B, Mahjoub Y, Desebbe O, et al. Clinical relevance of pulse pressure variations for predicting fluid responsiveness in mechanically ventilated intensive care unit patients: the grey zone approach. Crit Care. 2014;18(6):587.

54. Chytra I, Pradl R, Bosman R, Pelnar P, Kasal E, Zidkova A. Esophageal Dopplerguided fluid management decreases blood lactate levels in multiple-trauma patients: a randomized controlled trial. Crit Care. 2007;11(1):R24.

Ready to submit your research? Choose BMC and benefit from:

- fast, convenient online submission

- thorough peer review by experienced researchers in your field

- rapid publication on acceptance

- support for research data, including large and complex data types

- gold Open Access which fosters wider collaboration and increased citations

- maximum visibility for your research: over $100 \mathrm{M}$ website views per year

At BMC, research is always in progress.

Learn more biomedcentral.com/submissions 